\title{
Modulation of Balance Between Apoptosis and Proliferation by Lipid Peroxidation (LPO) During Rat Liver Regeneration
}

\author{
María Teresa Ronco, ${ }^{1}$ María de Luján Alvarez, ${ }^{1}$ Juan Monti, $^{1}$ María Cristina Carrillo, ${ }^{1}$ \\ Gerardo Pisani, ${ }^{2}$ María Cristina Lugano, $^{2}$ and Cristina E. Carnovale ${ }^{1}$ \\ ${ }^{1}$ Instituto de Fisiología Experimental, Consejo Nacional de Investigaciones Científicas y Técnicas \\ (CONICET), Facultad de Ciencias Bioquímicas y Farmacéuticas (Universidad Nacional de \\ Rosario), Argentina \\ ${ }^{2}$ Area de Morfología, Facultad de Ciencias Bioquímicas y Farmacéuticas (Universidad Nacional de \\ Rosario), Argentina
}

Accepted October 9, 2002

\begin{abstract}
Background: This work aims to investigate the role of lipid peroxidation (LPO) at early stages of liver regeneration and to evaluate the balance between apoptosis and cell proliferation during this process.

Methods: Sham and partial hepatectomized (PH) male Wistar rats were randomized in seven groups: Control (untreated), E-Control (injected with vitamin E-vehicle), C-Control (injected with vitamin C-vehicle), El (vitamin E $100 \mathrm{mg} / \mathrm{kg}$ body weight), E2 (vitamin E $600 \mathrm{mg} / \mathrm{kg}$ body weight), Cl (vitamin C $30 \mathrm{mg} / \mathrm{kg}$ body weight), C2 (vitamin C $100 \mathrm{mg} / \mathrm{kg}$ body weight).

Results: Vitamin treatments attenuated the increase of LPO level observed in total homogenate and microsomes at 3 and $5 \mathrm{hr}$ after PH. Both antioxidant vitamins attenuated the increase in Bax pro-apoptotic protein and augmented $\mathrm{Bcl}-\mathrm{xL}$ antiapoptotic protein levels $(35 \%)$ at
\end{abstract}

3 and $5 \mathrm{hr}$ post-PH; Bcl-xL/Bax ratio was, therefore, increased. A direct linear relationship between LPO levels and Bax mitochondrial protein levels was seen. Vitamin-treatments diminished the apoptosis index with respect to $\mathrm{PH}$-Control values, so that this parameter showed a linear relationship with LPO levels. At $24 \mathrm{hr}$ after $\mathrm{PH}$, the vitamin treatments increased the peak of $\left[{ }^{3} \mathrm{H}\right]$-thymidine incorporation into DNA and the proliferative index (PI), measured as PCNA expression; an inverse relationship between PI and LPO levels could be demonstrated.

Conclusion: Our data show that the diminution of LPO levels by vitamin-treatment post-PH produces both an attenuation of cellular apoptosis and a marked increase in the proliferation process, suggesting that the modulation of LPO has a role in liver regeneration process.

\section{Introduction}

Most hepatocytes in adult liver are quiescent with respect to proliferation. However, following a twothirds hepatectomy, liver switches to a proliferative state and reenters the cell cycle $(1,2)$. The damage caused by surgical resection results in a cascade of growth factor and cytokine induction designed to restore the liver mass to its original size $(3,4)$. Cell proliferation begins very early during rat liver regeneration, peaking for hepatocytes at $24 \mathrm{hr}$, with a smaller further peak between 36 and $48 \mathrm{hr}$. Finally, the remnant liver undergoes almost complete restoration of the lost mass and function by about 1 week $(5,6)$. Partial hepatectomy is the most often used model to study liver

Address correspondence and reprint requests to: Cristina E. Carnovale, Instituto de Fisiología Experimental, Consejo Nacional de Investigaciones Científicas y Técnicas (CONICET), Facultad de Ciencias Bioquímicas y Farmacéuticas Universidad Nacional de Rosario, Suipacha 570, 2000-Rosario, Argentina. Fax: (54)341-4399473; e-mail: ifise1@citynet.net.ar regeneration and the molecular signaling and factors involved in cell proliferation.

The determinants of hepatocyte proliferation during liver regeneration are highly complex, and different mechanisms operate during initiation of DNA synthesis and the termination of proliferative surge (7). The control of liver regeneration and the events involved in regulating the growth of the organ remain unknown. It has become increasingly apparent that apoptosis plays a key role in the cell cycle (8). Many proteins that can induce cell death are, in fact, components of the cell division cycle (9). Apoptosis is an important process by which organisms are able to eliminate unwanted, senescent, or damaged cells in absence of an inflammatory response. It plays a critical role in several physiologic as well as pathophysiologic processes $(10,11)$. The cellular threshold for apoptosis is highly regulated, especially by members of the Bcl-2 protein family. Members of this family are anti-apoptotic proteins (Bcl-2, Bcl-xL, Bcl-w) while others can promote programmed cell death (Bax, Bak, Bad, Bcl-xS) $(9,10)$. 
In a previous work, we found that after partial hepatectomy an increase in lipid peroxidation (LPO) level in subcellular fractions of rat liver occurs (12). It is known that this increase in LPO might be a modulator of cell division, influencing both the initiation and cessation of mitosis in the regenerating liver (13). LPO is one of the cellular pathways involved in oxidative damage. Oxidative stress, which is an excessive production of reactive oxygen species (ROS), can damage cells by triggering LPO and by altering protein and nuclear acid structures $(14,15)$. ROS is an autocatalytic mechanism that can lead to program cell death (apoptosis) $(16,17)$. To prevent oxidative damage and allow survival in an oxygen environment mammalian cells have developed an elaborate antioxidant defense system that includes enzymatic activity, as with glutathione reductase, catalase, and peroxidase, as well as nonenzymatic antioxidants, such as glutathione, vitamin C, and vitamin E. Antioxidant vitamins act as scavenging oxygen free radicals, preventing formation of high reactive lipid peroxidation (18-21).

The precise role that LPO plays in the process of hepatic regeneration is not known as yet. In the present study, we tested whether the modification of LPO level, using vitamin treatments, is implicated in the expression of pro-apoptotic and antiapoptotic proteins during liver regeneration after $70 \%$ partial hepatectomy. We also investigated the role of LPO in the balance between apoptosis and cell proliferation.

\section{Materials and Methods}

\section{Animals and Surgical Procedures}

Male Wistar rats weighing 360-400 g were housed two per cage and maintained at 12-hr light/dark period. Rats were fed ad libitum with a normal standard diet and water. All the experimental protocols were performed according to the Guide for the Care and Use of Laboratory Animals (National Institutes of Health, Publication no. 86-23, revised 1985). Sham operated animals (Sh) were laparotomized under pentobarbital anesthesia ( $50 \mathrm{mg} / \mathrm{kg}$ body weight, IP) to provide a control for surgical conditions. Twothirds hepatectomy $(\mathrm{PH})$ consisted of removal of the central and left lateral lobes as originally described by Higgins and Anderson (22). Animals were killed $3,5,24$, and $48 \mathrm{hr}$ after surgery. To avoid variations due to circadian rhythm, animals were always sacrificed at the same time day (between 10:00 and 12:00 hr).

At time of sacrifice, all rats were bled through a cardiac puncture after pentobarbital anesthesia and liver were removed. Pieces of liver tissue were processed for immunohistochemical studies. The remaining liver tissue was frozen in liquid nitrogen and stored at $-70^{\circ} \mathrm{C}$ until the analytical assays.

\section{Modification of Cellular Antioxidant Levels}

To enhance cellular antioxidant levels we used two antioxidants: vitamin E ( $\alpha$-tocopherol) and vitamin $\mathrm{C}$ (ascorbic acid). Sh and $\mathrm{PH}$ were randomized in seven groups:

1. I-Control (untreated)

2. II-Injected subcutaneously with vegetal oil, the vitamin $\mathrm{E}$ vehicle (E-Control)

3. III-Injected IP with saline solution, the vitamin $C$ vehicle (C-Control)

4. IV and V-received subcutaneously vitamin E ( $\alpha$-tocopherol $0.95 \mathrm{~g} / \mathrm{ml}$ [Sigma Chemicals Co. St. Louis, MO, USA]) $100 \mathrm{mg} / \mathrm{kg}$ body weight (E1) and $600 \mathrm{mg} / \mathrm{kg}$ body weight (E2), respectively. Each dose was administrated $24 \mathrm{hr}$ before surgery (23).

5. VI and VII-injected IP with vitamin C (ascorbic acid, Sigma Chemicals Co), $30 \mathrm{mg} / \mathrm{kg}$ body weight (Cl) and $100 \mathrm{mg} / \mathrm{kg}$ body weight (C2). Vitamin $C$ solution was administrated $30 \mathrm{~min}$ before surgery (24).

\section{Assays for Lipid Peroxidation}

The amount of aldehydic product generated by lipid peroxidation in subcellular fractions was quantified by the thiobarbituric acid (TBA) reaction according to the method of Ohkawa et al. (25). The amount of TBA reactants (TBARS) was expressed in terms of malondialdehyde using 1,1,3,3-tetra methoxypropane as standard.

Estimation of lipid peroxidation-related dienes conjugation was made in microsomal fraction essentially as described by Recknagel and Glende (26).

Protein content in each subcellular fraction was measured by the method of Lowry et al. (27), using human serum albumin as standard.

\section{Western Blot Analysis}

Liver tissue lysates were prepared by homogenization of frozen tissues in 3 volumes of lysating RIPA buffer containing PBS, $1 \%$ Triton, $0.5 \%$ sodium deoxycholate, $0.1 \%$ SDS, 1 mM phenylmethylsulfonyl fluoride, $10 \mu \mathrm{g} / \mathrm{ml}$ leupeptin, and $1 \mu \mathrm{g} / \mathrm{ml}$ aprotinin. After $30 \mathrm{~min}$ of incubation at $0^{\circ} \mathrm{C}$ and three freezethaw cycles, lysates were cleared by centrifugation at $15,000 \mathrm{rpm}$ for $30 \mathrm{~min}$, and supernatants were kept at $-70^{\circ} \mathrm{C}$. For the preparation of mitochondriaenriched fractions, liver tissues were homogenized in 4 volumes of $300 \mathrm{mmol} / \mathrm{L}$ sucrose with protease inhibitors. Homogenates were centrifuged at $1000 \mathrm{~g}$ to remove unbroken cells, nuclei, and heavy membranes. Mitochondria fractions were then obtained by centrifugation at $3000 \mathrm{~g}$ at $4^{\circ} \mathrm{C}$ for $15 \mathrm{~min}(28)$. For Western blot analysis, aliquots of whole cell or mitochondria-enriched protein (50 $\mu \mathrm{g}$ per lane) were subjected to $12 \%$ SDS-PAGE and transferred to polyvinyl difluoride membranes (Perkin Elmer 
Life Sciences, Boston, MA, USA). After blocking, blots were incubated overnight at $4^{\circ} \mathrm{C}$ with either anti-mouse Bax or anti-human Bcl-xL antibodies (1:500, Santa Cruz Biotechnology, Santa Cruz, CA, USA). The membranes were then incubated with either anti-rabbit or anti-mouse Ig G-peroxidase conjugates (1:5000 dilution, Amersham Life Science) and bands were detected by enhanced chemiluminescence detection (Amersham Pharmacia Biotech, Little Chalfont, Buckinghamshire, England). Autoradiographs were obtained by exposing polyvinyl difluoride membranes to Kodak XAR film, and the bands were quantitated by densitometry (Shimadzu CS-9000).

\section{Immunohistochemistry}

Liver slices from different lobes were fixed in $10 \%$ $\mathrm{v} / \mathrm{v}$ formalin solution and embedded in low-melting paraffin. Sections of slides were deparaffinized in xylene and alcohol and then rehydrated in deionized water. Immunohistochemical staining procedures were then performed (29).

Determination of Apoptotic Index Quantitative analysis of apoptosis was performed by in situ specific labeling of fragmented DNA using a modified terminal deoxynucleotidyl transferase (Tdt) mediated biotin-deoxyuridine triphosphate nick-end labeling (TUNEL) method (30) (Promega, Madison, WI, USA). Apoptotic cells were counted only if they were TUNEL positive and displayed hallmark characteristics of apoptosis. An apoptotic index (AI) was calculated for each sample by counting the number of positively stained hepatocyte nuclei divided by the total number of hepatocytes and expressed as percentage. The number of apoptotic hepatocytes was assessed by systematically scoring at least 6000 hepatocytes per slide at a magnification of $400 \times$.

Determination of Proliferative Index Sections of slides were examined by immunohistochemical staining with anti-proliferating cell nuclear antigen (PCNA) antibodies 24 and $48 \mathrm{hr}$ after surgery. PCNA was visualized by the method of Greenwell et al. (31) using anti-PCNA from Santa Cruz Biotechnology as primary antibody. Ten random fields of liver sections (400 $\times$ magnification) were evaluated and scored. All PCNA positive cells in G1, S, M, and G2 phases were judged as proliferating cells and scored per 1000 hepatocytes.

\section{DNA Synthesis}

Twenty-four and $48 \mathrm{hr}$ after the surgery $\left[{ }^{3} \mathrm{H}\right]$ thymidine (New Life Sciences, Boston, MA, USA) $(10 \mu \mathrm{Ci} /$ 200 g body weight.) was injected IP, and rats were sacrificed $1 \mathrm{hr}$ after injection. Liver tissues were immediately excised and washed with ice-cold physiologic saline. The incorporation of radioactivity into the acid-insoluble fraction was measured by a Liquid Scintillation Counter (1214 Rack Beta, Pharmacia, Wallac OY, Finland). The incorporation was expressed as dpm/mg DNA (32).

\section{Regression Coefficient Values}

From data shown in Figures 1 and 2, a direct linear relationship was obtained between Bax mitochondrial protein levels and LPO of total homogenates $(r=0.70 ; p<0.0046 ; n=14)$. In the same way, a direct linear relationship between AI and LPO of total homogenates was obtained $(r=0.70 ; p<0.007$; $n=14)$. Regarding PI, an inverse linear regression was seen $(r=-0.74, p<0.0046 ; n=14)$.

\section{Statistical Analysis}

Results were expressed as mean \pm SE. Significance in differences was tested by one-way ANOVA, followed by Tukey's test to determine difference between groups. Differences were considered significant when the $p$ value was $<0.05$. Correlations were examined by Spearman rank linear regression.

\section{Results}

\section{Lipid Peroxidation and Antioxidant Administration}

LPO was measured in total homogenate and in microsomal fraction obtained by differential centrifugation. Total homogenate and microsomal fraction from 3- and 5-hr PH-Control rats showed a significant increase of TBARS level as compared with their Sh-Control (Fig. 1).

To elucidate whether changes in LPO levels were associated with early steps of the liver regeneration process, we treated the animals with two antioxidants: $\alpha$-tocopherol (lipophilic antioxidant) or ascorbic acid (reducing agent in aqueous phase). The vehicle treatment of $\mathrm{Sh}$ and $\mathrm{PH}$ animals (E-Control and C-Control) showed no difference relative to $\mathrm{Sh}$ and $\mathrm{PH}$-Control, respectively (data not shown); all results were analyzed with respect to Control animals.

Both antioxidants at two different doses (E1, E2, $\mathrm{C} 1, \mathrm{C} 2)$ attenuated the increase of TBARS levels in both total homogenate and microsomal fractions after $\mathrm{PH}$ at the studied times (Fig. 1). Sh-vitamin treated groups showed no statistically significant changes in TBARS levels compared to Sh-Control group at any time tested. The variation in LPO levels in the microsomal fraction, measured as conjugated dienes, was similar to those observed in TBARS level (Table 1).

\section{Bax-Bcl-xL Protein Levels}

To determine the balance between apoptosis and cell proliferation we examined the expression of Bax and Bcl-xL proteins by western blot analysis at 3 and $5 \mathrm{hr}$ post-PH. Bax and Bcl-xL are members 

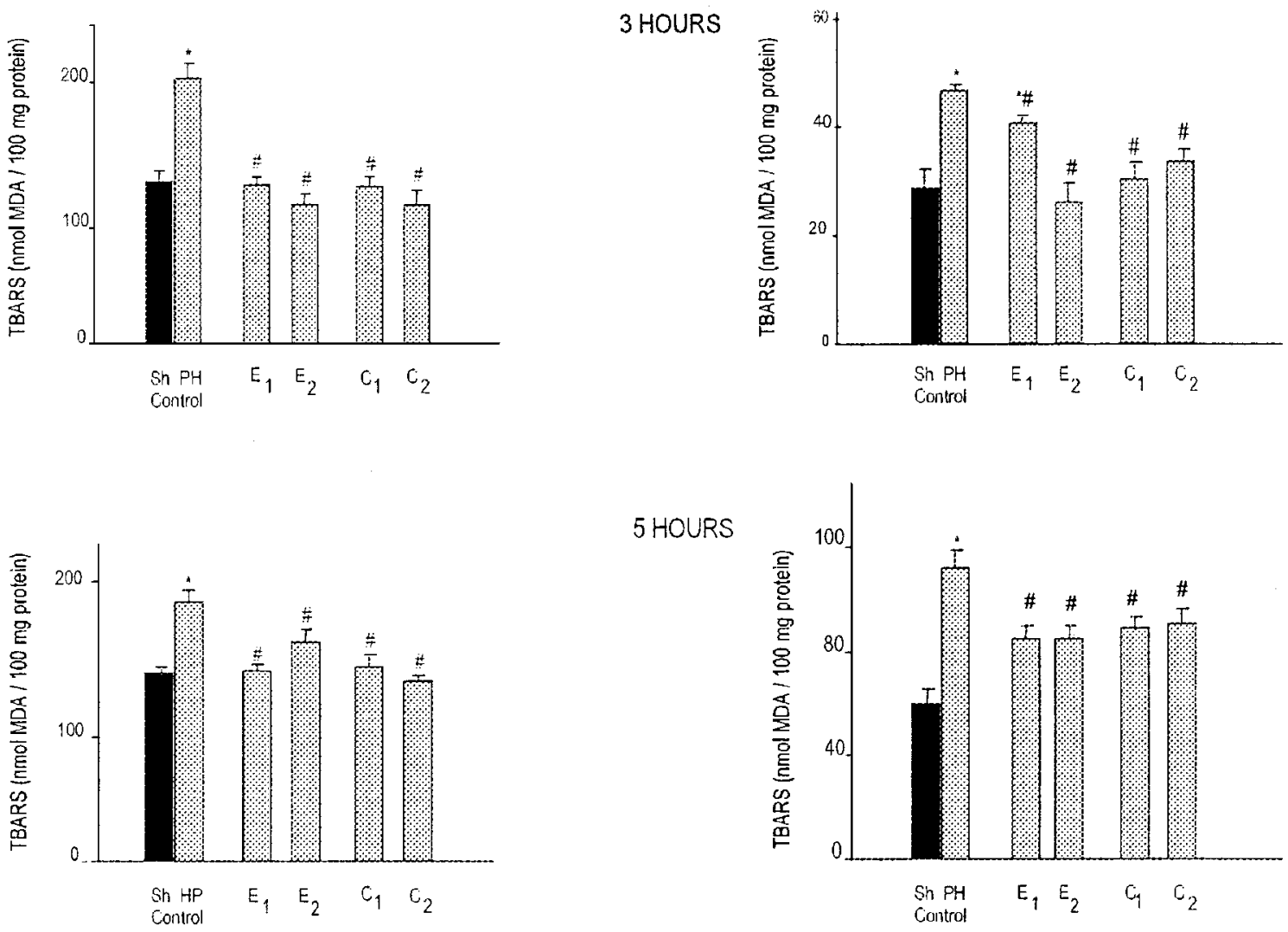

Fig. 1. Lipid peroxidative activity in hepatic homogenate and microsomal fraction in Sh (black bars) and PH (gray bars) with and without vitamin-treatment. Control: Group of animals without treatment; vitamin E: El (30 mg/kg body weight), E2 (600 mg/kg body weight); and vitamin C: C1 (30 mg/kg body weight), C2(100 mg/kg body weight). The levels of TBARS (nmol of $\mathrm{MDA} / \mathrm{mg}$ protein) were determined in the homogenate and microsomes 3 and $5 \mathrm{hr}$ after surgery. Results are expressed as mean \pm SE for at least six observations. Sh vitamin-treated show no statistically significant changes with respect to Sh-Control group at all times tested. ${ }^{*} p<0.05$ versus Sh. \#Statistically significant versus PH-Control.

of the Bcl-2 protein family, which plays a major role as regulator of the apoptotic process: whereas Bax promotes apoptosis, Bcl-xL protects cells from programmed cell death (10).

Bax protein levels in total lysates showed an increase in PH-Control animals with respect to $\mathrm{Sh}$, at 3 and $5 \mathrm{hr}$ after PH (data not shown). To determine whether enhanced expression of Bax protein after PH was associated with an increase in the mitochondrial translocation, we analyzed the expression of this protein in liver mitochondrial fraction. In Control group, Bax showed an increase of $48 \%$ in the liver mitochondrial fraction after $\mathrm{PH}$, relative to Sh animals (Fig. 2). Vitamin-treatments reduced the level of Bax protein by $34 \%$ for $\mathrm{PH}-\mathrm{E}$ and $30 \%$ for $\mathrm{PH}-\mathrm{C}$ as compared with $\mathrm{PH}$-Control group (Fig. 3).

Three and $5 \mathrm{hr}$ after $\mathrm{PH}$, mitochondrial Bcl-xL levels in Control animals showed an increase of $50 \%$ relative to corresponding Sh (Fig. 2). Vitamin- treatments increased Bcl-xL levels (35\%) in $\mathrm{PH}$ animals versus $\mathrm{PH}-$ Control values (Fig. 4) In addition, $\mathrm{Bcl}-\mathrm{xL} / \mathrm{Bax}$ mitochondrial protein ratios were calculated (Table 2). The Bcl-xL/Bax protein ratios were significantly elevated $(p<0.05)$ in all vitamin treated $\mathrm{PH}$ rats.

Expression of Bax and Bcl-xL proteins did not show differences between vehicle treated-PH animals and PH-Control (data not shown), so all the results were analyzed with respect to Control-PH group.

\section{Immunohistochemical Analysis}

Apoptotic Index Table 3 shows the AI in each experimental group. At 3 and $5 \mathrm{hr}$ after $\mathrm{PH}$, apoptosis was induced in Control group. Vitamin-treatments decreased the number of apoptotic cells in $\mathrm{PH}-$ rats versus Sham values.

Proliferative Index PCNA staining allows differential identification of cells in the late G1, S, G2, 


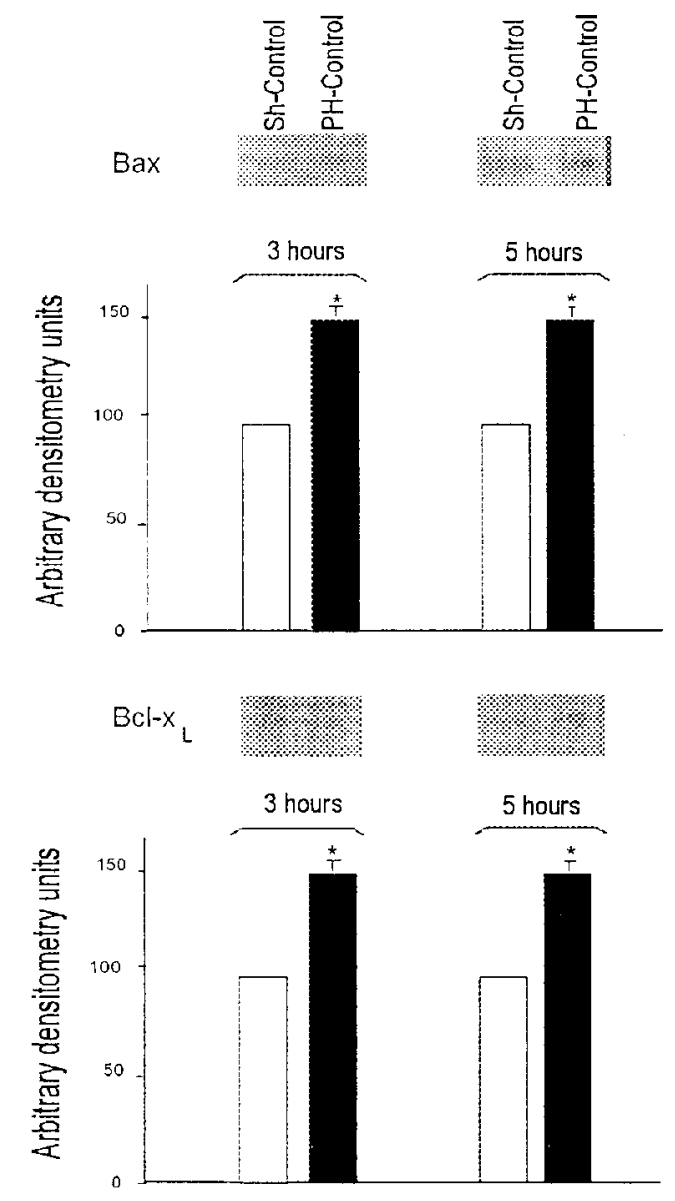

Fig. 2. Immunoblot analysis of Bax and Bcl-xL protein expression in liver mitochondrial fraction at 3 and 5 hr post PH. Lanes 1 and 3: Sh-Control. Lanes 2 and 4: PH-Control. The accompanying bars represent the densitometry expressed in percent from five separate animals sets. Bax levels in $\mathrm{PH}$-Control were increased $48 \%$ with respect to Sh-Control. Bcl-xL levels in $\mathrm{PH}$-Control were increased $50 \%$ with respect to Sh-Control. *Statistically significant against Sh-Control.

and mitotic phases. As expected, $24 \mathrm{hr}$ after surgery PI of PH-Control was higher than ShControl. On the other hand, PI showed an increase in treated-vitamin animals with respect to PH-Control group (Table 4). PI decreased $48 \mathrm{hr}$ after $\mathrm{PH}$, although maintaining differences with respect to Control animals (data not shown). Figure 5 shows light microscope images from Sh-Control and hepatectomized with and without vitamins treatment $24 \mathrm{hr}$ after the surgery.

\section{DNA Synthesis.}

The regenerative process following $\mathrm{PH}$ was monitored by the time-course of $\left[{ }^{3} \mathrm{H}\right]$ thymidine incorporation into DNA in the remnant tissue. Figure 6 shows the rates of DNA synthesis $24 \mathrm{hr}$ after PH. The treatment with E1 increased the peak $182 \%$, E2 $350 \%$ and with two doses of ascorbic acid the increase was about $30 \%$. The peak decreased toward
Table 1. Microsomal conjugated dienos contens

\begin{tabular}{lcc}
\hline & \multicolumn{2}{c}{ Time after surgery $(\mathbf{h})$} \\
\cline { 2 - 3 } & \multicolumn{2}{c}{3} \\
\hline Sh-Control & $0.488 \pm 0.051$ & $0.497 \pm 0.040$ \\
$\mathrm{PH}-$ control & $0.800 \pm 0.080 *$ & $0.965 \pm 0.090^{*}$ \\
$\mathrm{PH}-\mathrm{E}_{1}$ & $0.704 \pm 0.010 \#$ & $0.400 \pm 0.050 \#$ \\
$\mathrm{PH}-\mathrm{C}_{1}$ & $0.623 \pm 0.090 \#$ & $0.436 \pm 0.010 \#$ \\
& & \\
\hline & & \\
Presence of microsomal conjugated dienes was detected by their \\
absorption at 233 nm. Sh-vitamin treated groups showed no \\
statistically significant changes with respect to Sh-Control group \\
at all times tested. Values are expressed as the mean \pm SE of at \\
least six animals per group. \\
*Significant difference versus Sh-Control $(p<0.05)$. \\
\#Significant difference versus PH-Control $(p<0.05)$.
\end{tabular}

$48 \mathrm{hr}$, although maintaining differences with respect to Control animals (data not shown). No significant differences exist between Sham-treated and ShamControl rats.

\section{Discussion}

In this work, we demonstrated that a diminution of LPO levels (via antioxidants treatments) is followed by an enhancement of cell proliferation, as indicated by an increased number of proliferating cells and a diminution in the AI.

Immediately after $\mathrm{PH}$, a sequential and regulated series of events begins as the quiescent hepatocytes progress in the cell cycle from G0 to Gl (33). The mechanisms regulating this process are complex and incompletely understood. As a consequence of liver damage or loss of liver mass, like that produced by a $70 \%$ partial hepatectomy, an ROS increase occurs $(19,20)$. Potentially toxic actions of free radicals are controlled by cellular antioxidants. In many studies, LPO was monitored as an indirect indicator of ROS generation $(13,14,19)$. Following PH, LPO levels were increased at all the periods of time studied, a finding consistent with our earlier observations (12).

Vitamin $\mathrm{E}$ is a lipid-soluble antioxidant that accumulates in cellular membranes and scavenges lipid-peroxil radicals, thus preventing the formation of highly reactive lipid peroxidation products (34). Similarly, vitamin $C$ is a potent scavenger of reactive species, particularly in plasma, cytosol, and other aqueous compartments (18). We selected these vitamins as a useful tool to increase cellular antioxidant defense; the hepatoprotective effects of vitamins on lipid oxidative damage are well established, and are partially attributed to their ability to scavenge oxygen free radicals $(18,35)$. We found 


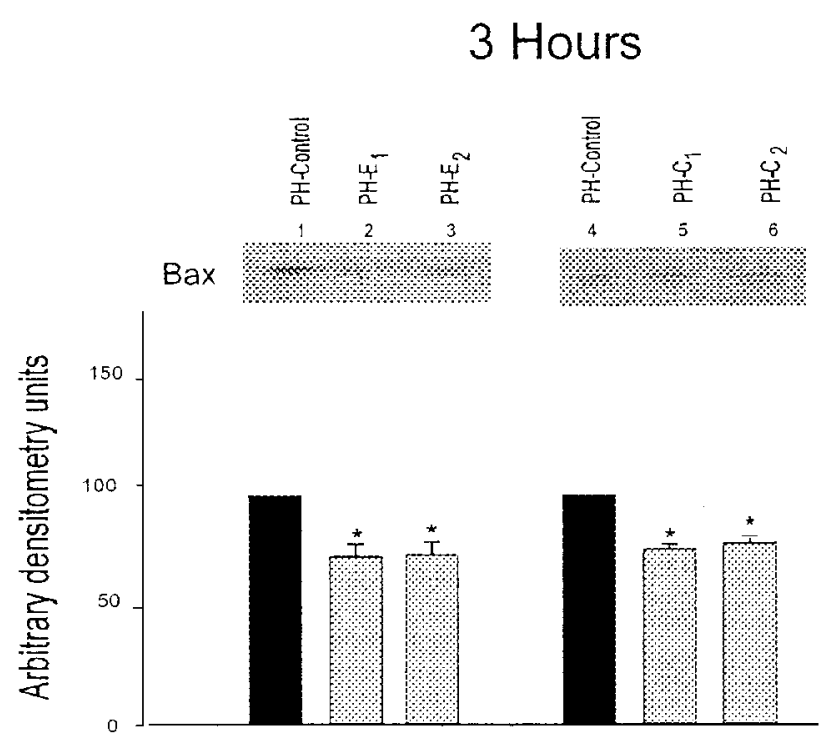

5 Hours

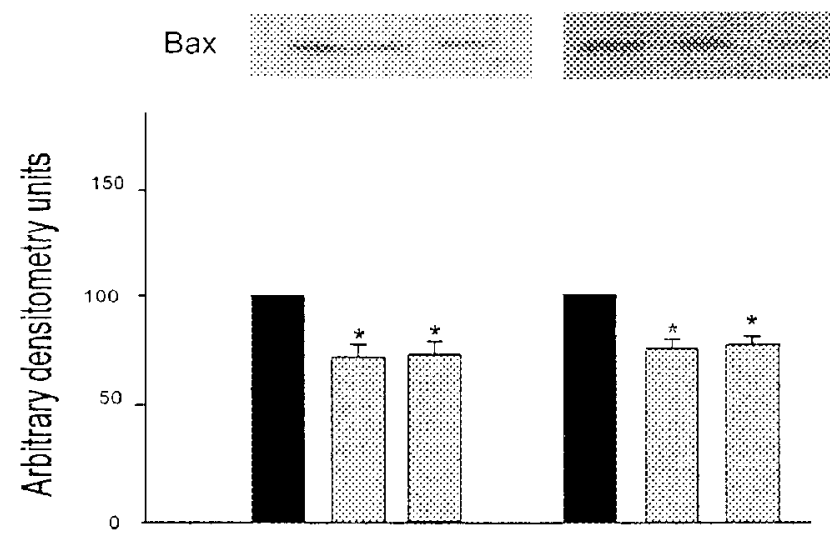

Fig. 3. Immunoblot analysis of Bax protein expression in liver mitochondrial fraction at 3 and $5 \mathrm{hr}$ post-surgery, with and without vitamin treatment. Lanes 1 and 4: $\mathrm{PH}-$ Control. Lane 2: PH-El (100 mg/kg body weight). Lane 3: PHE2 (600 mg/kg body weight). Lane 5: PH-Cl (30 mg/kg body weight). Lane 6: PH-C2 (100 mg/kg body weight). The accompanying bars represent the densitometry expressed in percent from five separate animals sets. $\mathrm{PH}$ vitamins-treated animals (gray bars) show a decrease of $30 \%$ in Bax protein respect to $\mathrm{PH}-$ Control (black bars) considering PH-Control as $100 \%$. *Statistically significant against PH-Control.

that the treatment with both antioxidants was able to protect the liver from oxidative stress. The neutralizing effect of vitamin-treatments against potent oxidizing species was apparent from the diminution of peroxidative decomposition of phospholipids in cellular membranes (LPO) observed at all times studied post-PH (Fig. 1).

ROS have been implicated in apoptosis (36). In liver, the involvement of reactive oxygen radicals has been suggested in apoptotic cell death of
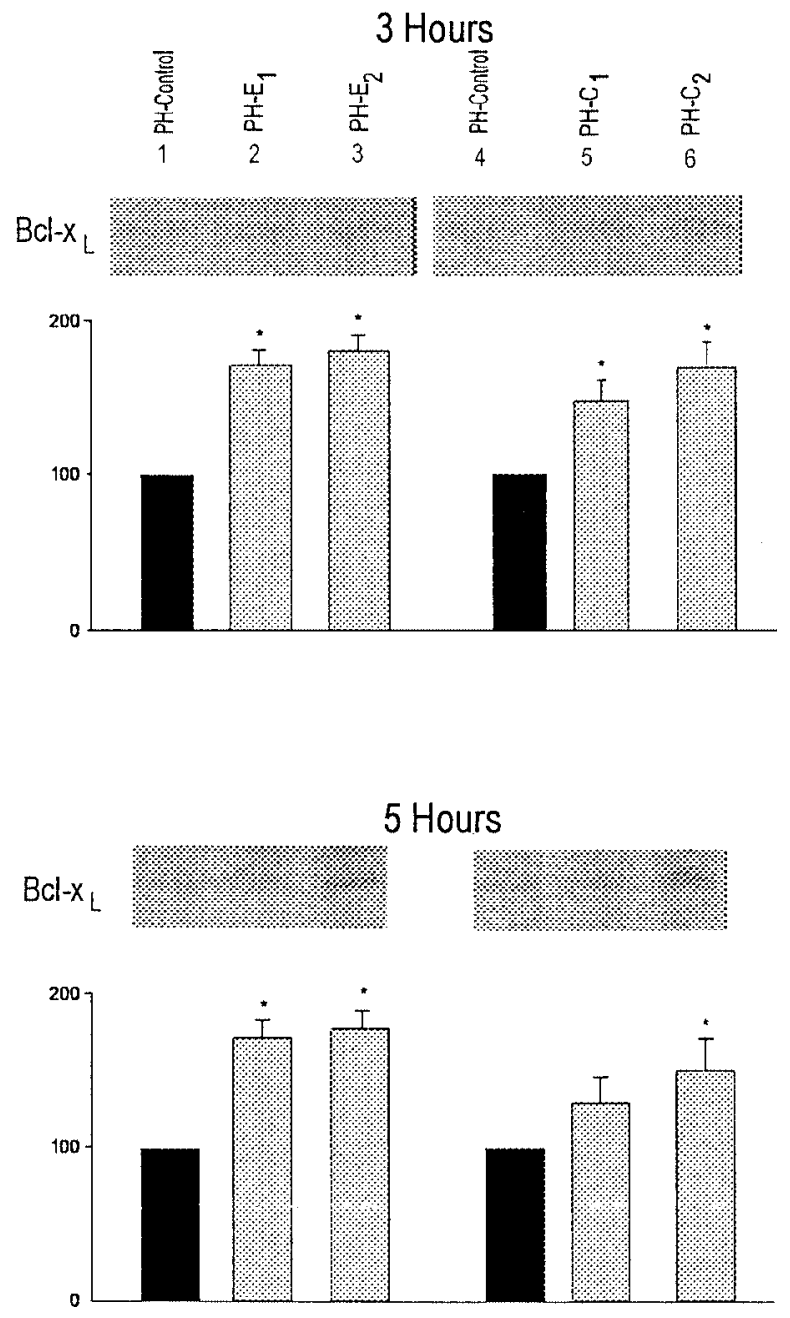

Fig. 4. Immunoblot analysis of $\mathrm{Bcl}-\mathrm{xL}$ protein expression in liver mitochondrial fraction at 3 and $5 \mathrm{hr}$ post-surgery. Lanes 1 and 4: PH-Control. Lane 2: PH-El (100 mg/kg body weight). Lane 3: PH-E2 (600 mg/kg body weight). Lane 5: PHC1 $(30 \mathrm{mg} / \mathrm{kg}$ body weight). Lane 6: PH-C2 (100 mg/kg body weight). Quantitation of immunoblot bands was performed by densitometry considering $\mathrm{PH}-\mathrm{Control}$ as $100 \%$. Results show the mean $\pm \mathrm{SE}$ of six rats. *Significant difference versus the corresponding PH-Control.

hepatocytes and endothelial cells (16). Bcl-xL and Bax are members of a growing family of genes that are involved in promoting either cell survival or cell death via apoptosis (10). Bax protein has been shown to promote cell death via homodimerization, whereas heterodimerization with Bcl-xL results in cell survival. Other authors have found an increase in basal levels of Bax protein and its RNA messenger (mRNA) in normal rats after PH (10). In accordance with their results, our findings indicate that during the first 3 and $5 \mathrm{hr}$ after $\mathrm{PH}$, Bax protein levels were increased (Fig. 2). Our results showed that oxidative process regulates protein levels of the Bcl-2 family. Administration of antioxidants reduced the pro-apoptotic protein (Bax) 
Table 2. Bcl- $x_{L} /$ Bax ratios

\begin{tabular}{cccccc}
\hline $\begin{array}{c}\text { Times after } \\
\text { surgery (hr) }\end{array}$ & PH-Control & PH-E & PH-E & PH-C & PH-C \\
\hline 3 & $1.2 \pm 0.2$ & $2.6 \pm 0.2^{*}$ & $2.7 \pm 0.1^{*}$ & $1.9 \pm 0.1^{*}$ & $1.9 \pm 0.1^{*}$ \\
5 & $1.1 \pm 0.2$ & $2.5 \pm 0.1^{*}$ & $2.5 \pm 0.1^{*}$ & $2.0 \pm 0.2^{*}$ & $1.8 \pm 0.1^{*}$ \\
\hline
\end{tabular}

Bcl- $\mathrm{x}_{\mathrm{L}}$ and Bax proteins were detected in liver mitochondrial fraction. Bcl- $\mathrm{x}_{\mathrm{L}} / \mathrm{Bax}$ ratio was calculated at 3 and 5 hrs post-PH. All values are expressed as mean $\pm \mathrm{SE}$ of six rats per group.

${ }^{*} p<0.05$ versus PH-Control.

level (Fig. 3) and increased the anti-apoptotic protein (Bcl-xL) level (Fig. 4). Furthermore, a direct linear relationship was obtained between Bax protein levels and total LPO levels in homogenate throughout the treatments. The relative prevalence of Bax and Bcl-xL protein are critical factors influencing cell fate; they promote cell either survival or death, whose ultimate outcome largely depends on the Bcl-xL/Bax ratio. This parameter increased with antioxidant-treatment both at 3 and $5 \mathrm{hr}$ after PH (Table 2).

Apoptosis is characterized by a number of morphologic features, including membrane blebbing, nuclear and cytoplasmic shrinkage, and chromatin condensation (11). In the present study, we used the TUNEL assay in combination with morphologic criteria to identify apoptotic cells. When these criteria were applied and hepatocytes were quantified, treatment with both antioxidants re-

Table 3. Apoptotic index (AI)

\begin{tabular}{lcc}
\hline & \multicolumn{2}{c}{ Time after surgery (h) } \\
\cline { 2 - 3 } & 3 & 5 \\
\hline Sh-Control & $1.0 \pm 0.1$ & $1.0 \pm 0.1$ \\
PH-Control & $3.0 \pm 0.5^{*}$ & $2.5 \pm 0.5^{*}$ \\
PH-E $_{1}$ & $1.0 \pm 0.1 \#$ & $1.0 \pm 0.1 \#$ \\
PH-C $_{1}$ & $1.0 \pm 0.1 \#$ & $1.0 \pm 0.1 \#$ \\
\end{tabular}

AI is expressed as percentage of apoptotic cells scored per 6000 hepatocytes per slide at magnification of $400 \times$. All values are expressed as mean \pm SE of six rats per group. Sh-vitamin treated groups showed no statistically significant changes respect to Sh-Control group at any time tested.

${ }^{*} p<0.05$ versus Sh-Control.

$\# p<0.05$ versus $\mathrm{PH}$-Control. duced AI (Table 3). Our results show a direct linear relationship between AI and total LPO levels, suggesting that apoptosis plays an important role in the process of hepatic remodeling during regeneration and in the fine tuning of the regenerative process. Therefore we hypothesize that a decrease in apoptotic protein levels with a concomitant decrease in apoptosis at early steps after $\mathrm{PH}$ are, in part, responsible for improvement in the proliferate response.

The processes of development and differentiation are regulated by a balance between proliferation, growth arrest, and programmed cell death (37). We found a significant increase in hepatocyte proliferation in resting liver after treatment with both antioxidants, as measured by PI (Table 4 and Fig. 5); this suggests that diminution of LPO levels enhances hepatocyte proliferation. This indicates a causal relationship between attenuated levels of LPO and stimulation of liver proliferation, supporting evidence that LPO inversely correlates with the rate of mitosis in several tissues (13). Furthermore, our results clearly show an increase in the peak of synthesis of DNA in hepatectomized rats when they were treated with each vitamin (Fig. 6).

The mechanism by which LPO modulates the proliferation/apoptosis balance cannot be completely ascertained from our results. LPO could influence some cellular processes in the proliferating organ. Free radicals and oxidative stress might initiate a general cell responsive mechanism and even activate transcriptional factors to serve as signal transducers between cytoplasm and nucleus, providing a framework supporting an important role of LPO during early steps of the liver regeneration (13).

Overall, the observations in this study support the role of LPO as an active participant in the regulation the balance between cellular proliferation and apoptosis after PH. Further studies are required to identify the precise mechanism(s) responsible for the protective effects of the antioxidant vitamins and also to elucidate how oxidative stress regulate liver regeneration after $\mathrm{PH}$. 
Table 4. Proliferative index

\begin{tabular}{cccccc}
\hline Sh-Control & PH-Control & PH-E & PH-E & PH-C $_{1}$ & PH-C $_{2}$ \\
\hline $3.3 \pm 1.3$ & $21.4 \pm 1.5^{*}$ & $36.2 \pm 3.5^{* \#}$ & $38.3 \pm 3.9^{* \#}$ & $32.3 \pm 0.5^{* \#}$ & $30.4 \pm 0.5^{* \#}$ \\
\hline
\end{tabular}

PI is expressed as proliferating hepatocytes cells per 1000 hepatocytes evaluated in 10 random fields of liver sections $(400 \times$ magnification). Sh-vitamin treated showed no statistically significant changes respect to Sh-Control group at all times tested. All values represent mean $\pm \mathrm{SE}$ of six animals per group.

${ }^{*} p<0.05$ versus Sh-Control.

$\# p<0.05$ versus $\mathrm{PH}-$ Control.
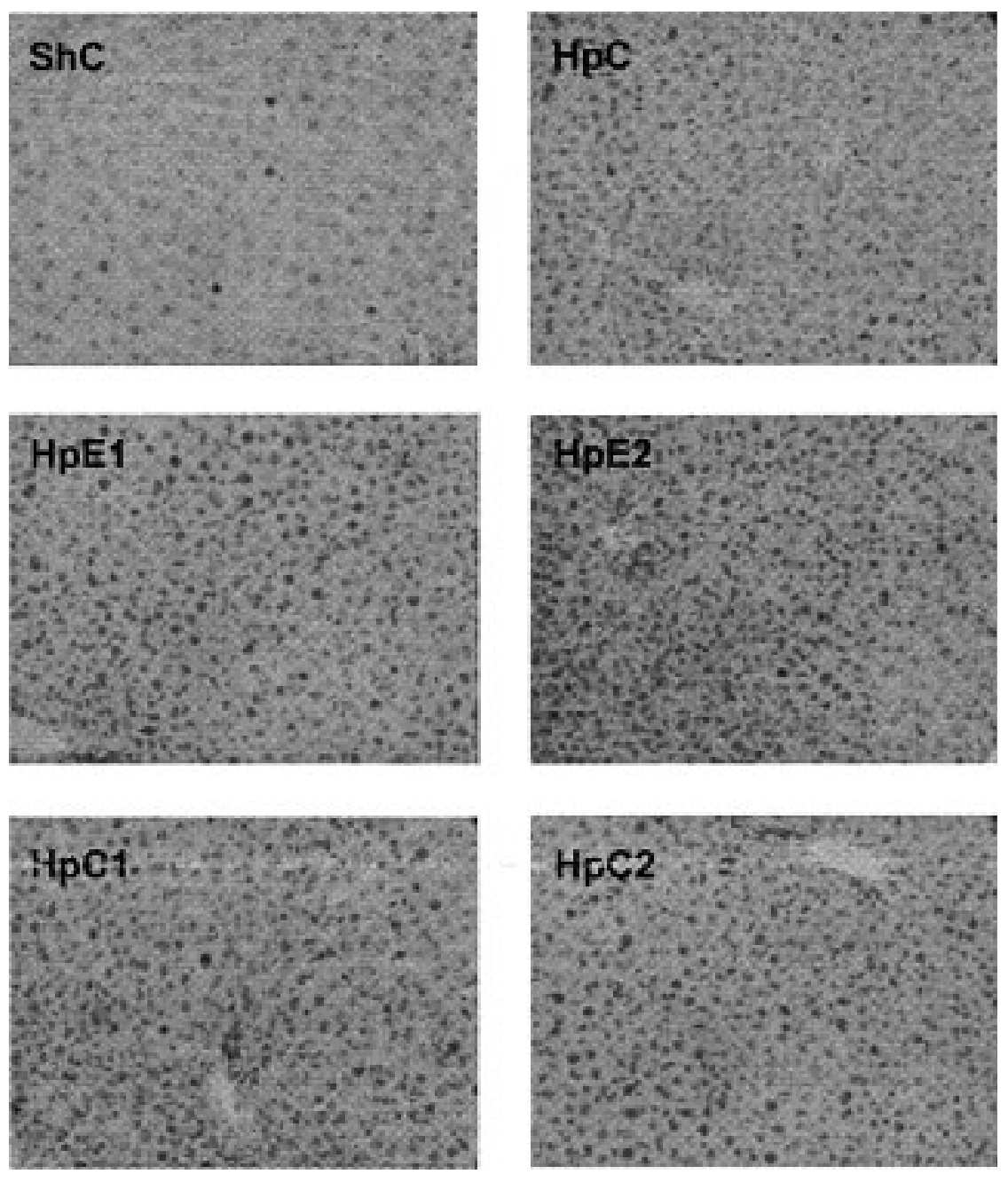

Fig. 5. Light microscope images of PCNA detection $24 \mathrm{hr}$ after surgery from (A) Sh-Control, (B) PH-Control, (C) PH-El (100 $\mathrm{mg} / \mathrm{kg}$ body weight), (D) PH-E2 (600 mg/kg body weight), (E) PH-C1 (30 mg/kg body weight), PH-C2 (100 mg/kg body weight). Six animals were studied in each group. Positive immunohistochemical staining for PCNA was evaluated by the brown to black reaction product that correlates with the different phases of the cell cycle. PI of PH-vitamin treated animals were higher than PH-Control animals. No difference was observed between Sh-Control, Sh-treated with vitamins or theirs corresponding vehicles. 


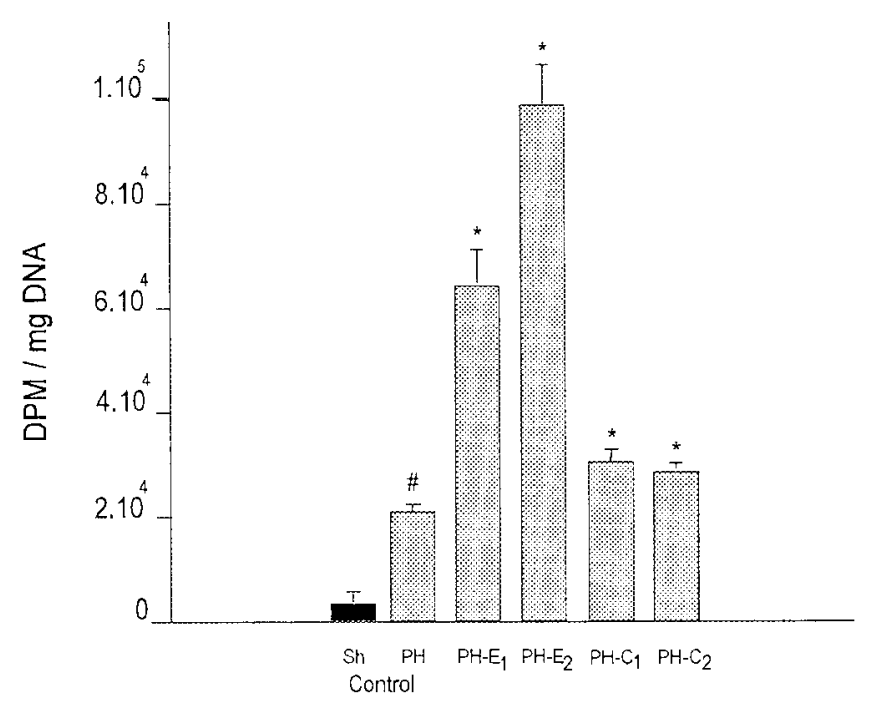

Fig. 6. Methyl $\left[{ }^{3} \mathrm{H}\right]$ thymidine incorporation to DNA $24 \mathrm{hr}$ after surgery in Sh-Control (black bar) and in PH rats (gray bars) with and without treatment. $\mathrm{PH}$-Control, vitamin $\mathrm{E}$ : E1 (dose $30 \mathrm{mg} / \mathrm{kg}$ body weight); E2 (dose $600 \mathrm{mg} / \mathrm{kg}$ body weight); and vitamin C: C1 (dose $30 \mathrm{mg} / \mathrm{kg}$ body weight); C2 (100 mg/kg body weight). (Sh-treated and Sh-Control group.) Data are expressed in DPM/mg DNA as mean of six rats \pm SE. \#Significant difference versus Sh-Control $(p<0.05)$.

*Significant difference versus PH-Control $(p<0.05)$.

\section{Acknowledgments}

We gratefully acknowledge Dr. José Pellegrino for helpful discussions and his critical appraisal of the manuscript.

This work was supported by research grants from "Ramón Carrillo-Arturo Oñativia" and from Consejo Nacional de Investigaciones Científicas y Tecnológicas (CONICET).

\section{References}

1. Li W, Liang X, Leu J, et al. (2001) Global changes in interleukin-6-dependent gene expression patterns in mouse livers after partial hepatectomy. Hepatology 33: 1377-1386.

2. Teruhiro N, Hllerbrand C, Behrns K, et al. (1998) NFkB prevents apoptosis and liver dysfunction during liver regeneration. J. Clin. Invest. 101: 802-811.

3. Nagy P, Teramoto T, Factor V, et al. (2001) Reconstitutive liver mass via cellular hypertrophy in the rat. Hepatology 33: 339-345.

4. Angelis R, Kovalovich K, Cressman D, et al. R. (2001) Normal liver regeneration in $\mathrm{p} 50 /$ nuclear factor $\mathrm{kbl}$ knockout mice. Hepatology 33: 915-924.

5. Satdarshan P, Monga S, Pediaditakis $P$, et al. (2001). Changes in wnt/b-catenin pathway during regulated growth in rat liver regeneration. Hepatology 33: 1098-1109.

6. Kountouras J, Boura P, Lygidakis N. (2001) Liver regeneration after partial hepatectomy. Hepatogastroenterology 48: 556-562.

7. Boulton R, Woodman A, Calnan D, et al. (1997) Nonparenchymal cells from regenerating rat liver generate interleukin-la and -lb: a mechanism of negative regulation of hepatocyte proliferation. Regulation of apoptosis-associated genes in the regenerating liver. Hepatology 26: 49-58.

8. Fan G, Krent B, Steer CJ. (1998) Regulation of apoptosisassociated genes in the regenerative liver. Semin. Liver. Dis. 18: 123-140.
9. Kren B, Trembley J, Krajewski S, et al. (1996) Modulation of apoptosis-associated a genes bcl-2, bcl-x, and bax during rat liver regeneration. Cell Growth Differ. 7: 1633-1642.

10. Tzung S, Fausto N, Hockenbery D. (1997) Expression of bcl-2 family during liver regeneration and identification of bcl$\mathrm{x}$ as a delayed early response gene. Am. J. Pathol. 150: 1985-1995.

11. Patel T, Steer C, Gores G. (1999) Apoptosis and the liver: a mechanism of disease, growth regulation, and carcinogenesis. Hepatology 30: 811-815.

12. Carnovale C, Scapini C, Alvarez L, et al. (2000) Nitric oxide release and enhancement of lipid peroxidation in regeneration rat liver. J. Hepatol. 32: 798-804.

13. Aguilar-Delfin I, Lopez-Barrera F, Hernández-Muñoz R. (1996) Selective enhancement of lipid peroxidation in plasma membrane in two experimental models for liver regeneration: partial hepatectomy and acute $\mathrm{CCl} 4$ administration. Hepatology 24: 657-662.

14. Shen H-M, Shi C-Y, Lee H-P, et al. (1994) Aflatoxin B1induced lipid peroxidation in rat liver. Toxicol. Appl. Pharmacol. 127: 145-150.

15. Pinkus R, Weiner L, Daniel V. (1997) Role of antioxidants and oxidants in the induction of ap-1, NFkB, and the glutation s-transferase gene expression. J. Biochem. Chem. 271: 13422-13429.

16. Jaeschke H. (2000) Reactive oxygen and mechanism of inflammatory liver injury. J. Gastroenterol. Hepatol. 15: 718-724.

17. Jones B, Lo C, Liu H, et al. (2000) Role of caspasas and NFkB signaling in hydrogen peroxide- and superoxide-induced hepatocyte apoptosis. Am. J. Physiol. Gastrointest. Liver Physiol. 278: G693-G699.

18. Masayasu I. (1999) Protective mechanism against reactive oxygen species. In Arias $\mathrm{M}$, et al. eds. The Liver Biology and Pathobiology, 3rd ed. New York; pp. 443-459.

19. Horvát M, Gonzalez-Cabello R, Blázovics A, et al. (2001) Effect of silibinin and vitamin $\mathrm{E}$ on restoration of cellular immune response after partial hepatectomy. J. Ethnopharmacol. 77: 227-232.

20. Factor V, Laskowska D, Rugaard JM, et al. (2000) Vitamin E reduces chromosomal damage and inhibits hepatic tumor formation in a transgenic mouse model. Proc. Natl. Acad. Sci. 7: 2193-2201.

21. Lizard G, Miguet C, Bessede G, et al. (2000) Impairment with various antioxidants of the loss of mitochondrial transmembrane potential and of the cytosolic release of cytochrome c occurring during 7-ketocholesterol-induced apoptosis. Free Radic. Biol. Med. 28: 743-753.

22. Higgins AR, Anderson RM. (1931) Experimental pathology of the liver restoration of the liver of the white rat following partial surgical removal. Arch. Pathol. 12: 186-202.

23. Knight ME, Roberts RJ. (1985) Tissue vitamin e levels in new born rabbits after pharmacologic dosing influence of dose, dosage form, and route of administration. Dev. Pharmacol. 8: 96-106.

24. Su H, Han C, Liu M, et al. (2000) Phosphine inducedoxidative damage in rats: attenuation by melatonin. Free Radic. Biol. Med. 8: 636-642.

25. Ohkawa H, Ohishi N, Magi K. (1979) Assay of lipid peroxides in animal tissues by thiobarbituric acid reaction. Anal. Biochem. 95: 352-358.

26. Recknagel RO, Glende EA. (1984) Spectrophotometric detection of lipid conjugated dienes. Methods Enzymol. 105: 331-337.

27. Lowry OH, Rosebrough NJ, Farr AL, Randall RJ. (1951) Protein measurement with the follin phenol reagent. J. Biol. Chem. 193: 265-275.

28. de Duve C, Pressman BC, Gianetto R, et al. (1995) Tissue fraction studies. Intracellular distribution of enzymes in rat liver tissue. Biochem. J. 60: 604-617.

29. Gordon G, Coleman W, Gisham J. (2000) Bax-mediated apoptosis in the livers of rats after partial hepatectomy in the retrorsine model of hepatocellular injury. Hepatology 32: 312- 320.

30. Gold R, Schmied M, Giegerich G, et al. (1994) Differentiation between cellular apoptosis and necrosis by the combined use 
of in situ tailing and nick translation techniques. Lab. Invest. 71: 219-225.

31. Greenwell A, Foley JF, Maronpot RP. (1991) An Enhancement method for immunohistochemical staining of proliferating cell nuclear antigen in archival rodent tissues. Cancer Lett. 59: $251-256$.

32. McIntosh E, Gauthier T, Pettigren N, et al. (1992) Liver regeneration and the effect of exogenous putrescine on regenerative activity after partial hepatectomy in cirrhotic rats. Hepatology 16: 1428-1433.

33. Limuro Y, Nishiura T, Hellerbrand C, et al. (1998) NFOEB prevents apoptosis and liver dysfunction during liver regeneration. J. Clin. Invest. 101: 802-811.
34. Sokol R, Devereaux M, Khandwala R, O'Brien K. (1993) Evidence for involvement of oxygen free radicals in bile acid toxicity to isolated rat hepatocytes. Hepatology 147: 869-881.

35. Ozaki M, Fuchinove S, Teraoka S, Ota K. (1995) The in vivo cytoprotection of ascorbic acid against ischemia/reoxygenation injury of rat liver. Arch. Biochem. Biophys. 318: 439-445.

36. Hompton M, Orrenius S. (1997) Dual regulation of caspase activity by hydrogen peroxide: implications for apoptosis. FEBS Lett. 414: 552-556.

37. Sanchez A, Alvarez A, Benito M, et al. (1997) Cycloheximide prevents apoptosis, reactive oxygen species production, and glutathione depletion induced by transforming growth factor $\alpha$ in fetal rat hepatocytes in primary culture. Hepatology 26: 935-943. 